\title{
An antioxidant approach directed to non-healing wounds activation - A case report study
} \author{
Francisco Pedro García-Fernández ${ }^{6}$ \\ ${ }^{1}$ Advance Practice Nurse in Complex Chronic Wounds, West Sanitary District, Almería, Spain \\ ${ }^{2}$ Department of Nursing, Dean of the Faculty of Health Sciences, University of Almería, Spain \\ ${ }^{3}$ Department of Health sciences, University of Jaén, Spain \\ ${ }^{4}$ School of Nursing and Physiotherapy, University of Lleida, Spain \\ ${ }^{5}$ Librarian of research support unit, West Hospital, Almería, Spain \\ ${ }^{6}$ Department of Nursing, Vice-Dean of the Faculty of Health Sciences. University of Jaén, Spain
}

Juan Francisco Jiménez-García ${ }^{1 *}$, Gabriel Aguilera-Manrique ${ }^{2}$, Laura Parra-Anguita ${ }^{3}$, Joan Enric Torra-Bou $^{4}$, Irene Villegas-Tripiana $^{5}$ and

\begin{abstract}
It is well known that oxidative stress is a pathologic condition that compromises wound evolution, contributing to inflammatory environment maintaining and wound healing stagnation. This condition specially affects to aged people and to patients with important comorbidities, whereby healing capabilities are significantly impaired. In this work, a new therapeutic approach through a new antioxidant technology with natural components has been applied, in order to manage the oxidative stress, in 31 patients with complex chronic wounds of different etiologies, attended to the Sanitary West District in Almería (Spain). Wound assessments were performed at 4 , 8 and 12 weeks and main endpoints included the evolution of wound healing (\% of healing rate and Resvech 2.0 score) and pain relief. The treatment with antioxidant dressing produced a significant decrease of RESVECH 2.0 of $37.28 \%, 58.52 \%$ and $67.8 \%$ at 4, 8 and 12 weeks respectively and during the 12 week follow-up, $16 / 31$ wounds totally healed (50\%). In addition, a decrease in pain and biofilm presence in wounds were observed. In conclusion, the analyzed antioxidant approach could be a new therapeutic strategy directed to overcome the inflammatory phase in complex wounds and allow the progression over the healing process.
\end{abstract}

\section{Introduction}

Chronic wounds are wounds physiologically impaired with a disruption in the wound healing cycle [1]. Most of them are characterized by their stagnation in the inflammatory phase that prevent the progression over the healing process [2]. One of the physiologic common features existing in chronic wounds is the presence of oxidative stress (OS) [3,4]. OS appears when reactive oxygen species (ROS) produced by inflammatory cells that are recruited to the wound, are not effectively managed. When a wound is produced, inflammatory cells, mainly neutrophils, generate ROS as a mechanism of defence from microorganisms and as signalling molecules to activate healing pathways [5,6]. While acute wounds can efficiently regulate ROS production, decreasing their levels along with the inflammatory phase progression, in non-healing wounds, it has been shown that an elevated amount of ROS is maintained. This fact mainly occurs because most of patients with chronic wounds suffer from comorbidities (diabetes, vascular diseases, aging process, obesity) that additionally increase ROS production and decrease physiologic antioxidant defences, giving rise to a situation of OS [7-12].

In this context, a strategy that could manage the excess of ROS, can significantly contribute to overcome the inflammatory phase, and help to wound progression over the healing process. This way, we detected an antioxidant technology $y^{*}$, indicated for the inflammatory phase of complex wounds. An evaluation of product feasibility, performance, and utility in daily practice has performed, in order to develop a practical guide for the local nursing community and decide its potential inclusion in the panel of available products for wound management [13].

Antioxidant treatments consist of a lyophilized absorbent matrix, made of a galactomannan from vegetal origin obtained from the seeds of the carob tree and a matrix hydration solution that contains curcumin and acetylcistein. The three components act synergistically giving the product a potent antioxidant activity $[14,15]$.

Here it is presented the research of a group of specialists in chronic wound care of Andalusia Public Health System (Spain), in charge of new technologies analysis, about the first natural dressing specifically directed to manage the excess of free radicals in wound environment during inflammatory phase, with the aim of improving healing process specially in non-healing wounds. ${ }^{*}$ Reoxcare (Reoxcare.com; Histocell, Spain)

${ }^{\star}$ Correspondence to: Juan Francisco Jiménez-García, Advance practice nurse in complex chronic wounds. West Sanitary District, Almería, Spain, Tel: 0034610088696; E-mail: juanfrajime@gmail.com

Key words: chronic wounds, non-healing wounds, oxidative stress, bioactive antioxidant dressing, wound bed preparation

Received: February 05, 2021; Accepted: February 18, 2021; Published: February 25,2021 


\section{Methods}

\section{Study design}

Patients with complex wounds were recruited at nine different primary care units in West Health District of Almeria (Spain) coordinated by a wound care specialist from April 2017 to January 2019.

Data collected from each patient included:

- Demographic characteristics (age and sex)

- Patient's clinical background (concurrent diseases, other medications)

- Initial description of the wound (etiology, duration, size, location, VAS score)

A cut-off of 12 weeks (or healing, if occurred before) was established. Wounds were assessed at weeks 4,8 and 12 to determine their evolution.

Main variables of evolution of wound healing included RESVECH 2.0 score, healing rate (\%) and pain (visual analog scale-VAS) $[16,17]$. A secondary variable studied the presence of biofilm in the wound bed:

- RESVECH 2.0 score is a useful and validated tool to assess wound evolution that takes into account six main parameters: size of the lesions, depth and involved tissues, wound margins, type of tissue in the wound bed, level of exudates, presence of signs of infection/ inflammation. The scale is scored numerically, with values ranging from 0 to 35 points. Both ends of the scale correspond to a healed or to the worst possible wound, respectively. Data collection sheets for RESVECH 2.0 have clear operational definitions for each item with a numerical value that should be assigned to each variable/condition.

- Wound healing rate was estimated following Lundeberg et al. formula [18]: \% wound healing rate $=$ initial area - area at different times / initial area $\times 100$

- Presence of biofilm was determined according to clinical identification algorithm developed by Metcalf et al. [19,20], indicating biofilm presence or absence at each time point for every wound.

\section{Study population}

The study included 31 adult patients ( $\geq 18$ years) presenting nonhealing wounds from different aetiologies, with the aim to activate the wounds that were stalled in the inflammatory phase.

Inclusion criteria included patients with wounds with torpid evolution which complained with established criteria by wound care specialists for referral:

- Wounds where bacterial burden have been managed adequately at least for two-four weeks without positive evolution.

- Wound evolution period is long enough to consider it as torpid wound.

- Wound presents around 6 weeks of evolution without an improvement in RESVECH 2.0 score.

- Wound presents more than 12 weeks of evolution without clear signs of improvement.

- Wound care specialists considered antioxidant bioactive dressing as an appropriate treatment for the wound, independently of their type or etiology.

Following exclusion criteria were considered:
- To be less than 18 years old

- Intolerance to any of the components of antioxidant dressing

- The patient cannot be followed up due to any circumstances

- Short life expectancy

- Wound care specialist after analyzing previous applied treatment protocol detects that it can be improved. In this case a recommendation for treatment improvement was performed to be followed by primary care nurse.

\section{Wound management}

During the treatment with antioxidant dressing, all patients were managed according to good standard of care. Briefly, a general protocol was established for wound management that included the following steps: 1 . Cleaning the wound with sterile physiological saline solution; 2 . Antiseptic (clorhexidine solution) application; 3. In case of the presence of non-viable tissues in the wound bed, a sharp debridement was performed to deep clean the wound as much as possible; 4 . In moderate and highly exudative wounds, a barrier system was applied in the perilesional skin that included barrier creams with zinc, emollient-hydrating creams or HOFA (hyper-oxygenated fatty acids), depending on the patient's skin condition; 5. Antioxidant bioactive dressing application as primary dressing in contact with wound bed; 6 . Cover primary dressing with a polyurethane foam with or without silicone adhesive border, depending of the wound and patient perilesional skin; 7. Apply compressive therapy if it is indicated.

For antioxidant technology application, instructions from product leaflet were followed. Briefly blister with galactomannan vegetal matrix was opened, hydration solution directly poured over the matrix and after around one minute to achieve complete matrix hydration, it was directly applied to the wound bed. Wound was covered with the polyurethane foam as secondary dressing for exudates management and to retain antioxidant primary dressing. Dressings was changed every 2-4 days, depending on the level of wound exudates. Antioxidant dressing is a sterile single use medical device class IIb.

\section{Stattistical analysis}

Descriptive results were presented as percentage for qualitative variables, and mean, standard deviation (SD), range, and median for quantitative variable. All statistical analyses were performed using GraphPad Prism 5 (GraphPad Software Inc., San Diego, California). Values were expressed as mean \pm standard deviation. Evolution over time was analysed using repeated measures ANOVA (Analysis of variance) and non-parametric Friedman test. Comparison between defined times were performed using Student's unpaired t-test. All $\mathrm{p}$ values were twotailed and $p$ values less than 0.05 were considered significant. Biofilm related statistics were performed using $\chi^{2}$ test to analyse contingency tables.

\section{Ethical aspects}

Study was approved by Ethics Committee (approval code 48/2016) from Andalusia Health System, which confirmed that the study complies with ethical principles according to Helsinki Declaration (2013) and International Codes. Before entering the clinical trial and following study protocol guidelines, all patients read the study information, had the opportunity to ask questions to the attending nurse, signed the informed consent, which ensured data confidentiality and offered the choice to withdraw. 


\section{Results}

A total of 31 patients were recruited from West Health District of Almeria (Spain) in different wound care units. Out of these, 21 (67.7\%) were men and $10(32.2 \%)$ were women. All patients were Caucasian with a mean age of $63.68 \pm 15.27$ years (range: $26-90$; median 62 years) (Table 1).

All patients suffered from hard-to-heal wounds with a mean evolution time of $11,97 \pm 15,27$ months (range 2-74 months). Most of patients had important comorbidities such as type II diabetes or venous disease with specific therapeutic treatments. Wounds were mainly located at lower limbs (83\%) and the following etiologies were included: venous leg ulcers (13), pressure ulcers (3), spider bite (2), arterial ulcer (2), calciphylaxis ulcer (2), burn (1), neuropathic diabetic foot ulcer (1), cat scratch at origin but turned to a complex wounds with 8 months of evolution (1), multicausal (1), tumoural wound (1), wound after cranioplasty (1), actinic keratosis (1), post-surgery wound (1) and pilonidal sinus (1) (Figure 1).

A representative profile of a patient of the study included the following characteristics: 64 year old male with comorbidities and underlying pathologies that hinder the normal healing progression with an important inflammatory component. The wound had 1 year of evolution, with an initial size around $20 \mathrm{~cm}^{2}$, and RESVECH 2.0 score of 17 . He had moderate pain (5/10 VAS scale) in wound area and presence of biofilm.

Overall, 31 wounds were treated with a mean dressing change of every 3 days (2-4 days). Evolution of the wound healing, assessed by RESVECH 2.0, showed significant differences over time, as determined by Friedman Test $(\mathrm{p}<0.0001)$. RESVECH 2.0 reduced $37.28 \%, 58.52 \%$ and $67.8 \%$ at 4,8 and 12 weeks respectively from the start of antioxidant dressing treatment (Figure 2). Similarly, percentage of wound healing rate significantly increased over time (Friedman test $\mathrm{p}<0.0001$ ), and was $40 \%, 63 \%$ and $71 \%$ at 4,8 and 12 weeks (Figure 3), respectively. Over the 12 weeks follow-up time 16/31 wounds totally healed (50\%)

Another primary endpoint was the analysis of pain during the treatment with antioxidant dressing by VAS scale. Measurements

Table 1. Patient demographics

\begin{tabular}{|c|c|}
\hline Mean age \pm standard deviation (years) & $63.68 \pm 15.93$ \\
\hline Range, (median) years & $26-90,(62)$ \\
\hline
\end{tabular}

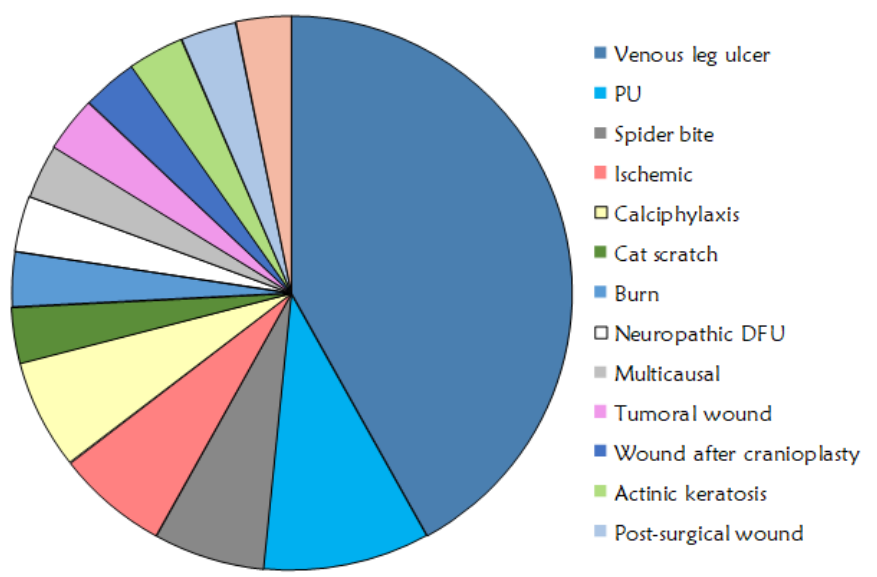

Figure 1. Etiologies of the wounds included in the study with bioactive antioxidant dressing

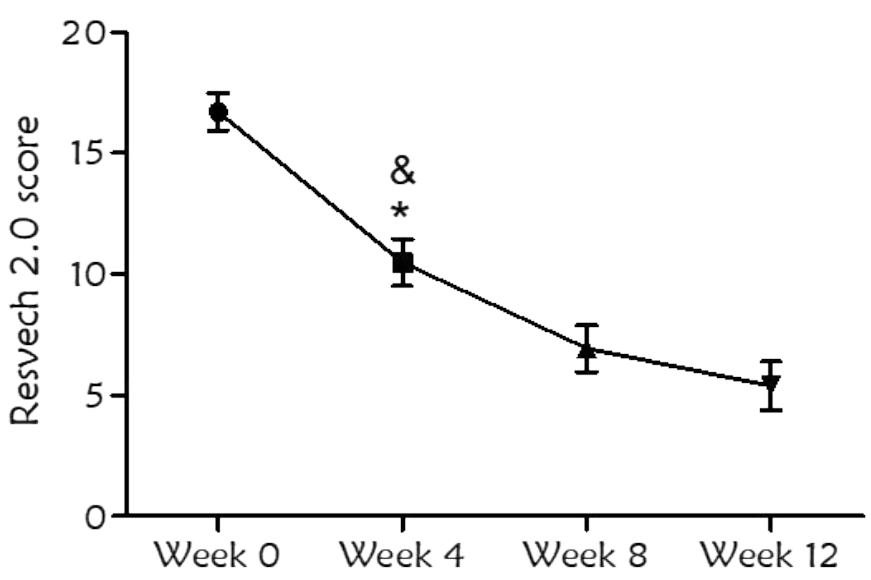

Figure 2. Evolution of wound healing during the study, assessed by RESVECH 2.0 scoring system (error bars represent $95 \%$ confidence interval). Statistical differences were found with respect to the start of the treatment at all timepoints (Friedman test, $\mathrm{p}<0.0001$ ). There were also differences when comparing week 4 with respect to week $8\left({ }^{*} \mathrm{p}<0.05\right)$ and week 4 with respect to week $12(\& \mathrm{p}<0.0001)$

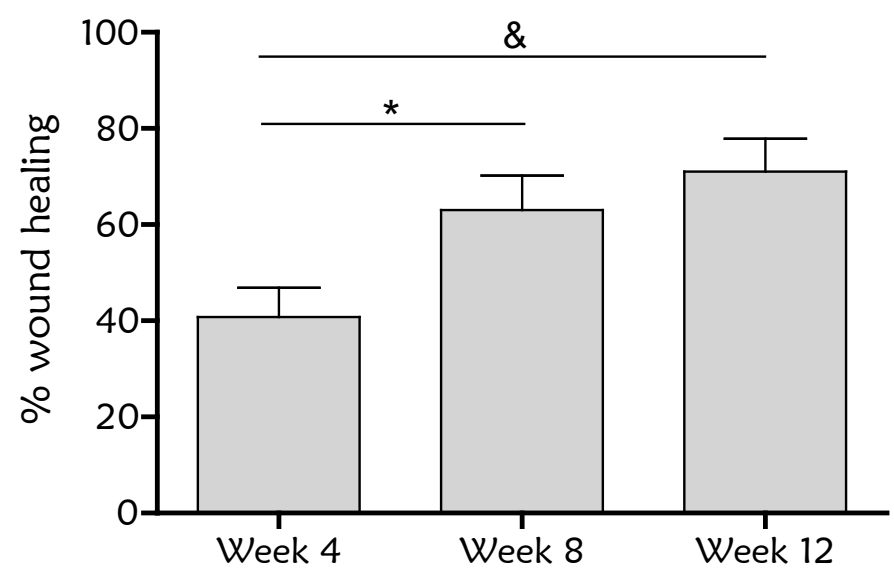

Figure 3. Percentage of wound healing over baseline during the treatment with antioxidant wound dressing. Statistical differences were found over baseline at all time points (One way ANOVA Friedman test, $\mathrm{p}<0.0001)$, and when compared week 4 with respect to week 8 $\left({ }^{*} \mathrm{p}<0.05\right)$ and week $12(\& \mathrm{p}<0.001)$

revealed a significant decrease in pain evolution (Friedman test $\mathrm{p}<0.0001$ ). There was a reduction of $43.8 \%, 66 \%$ and $77 \%$ over baseline at 4,8 and 12 week respectively. Additionally, there was total pain relief in $77 \%$ of the wounds at the end of the study (Figure 4).

In addition, there was a reduction of the presence of biofilm during the treatment with Reoxcare. Number of wounds with biofilm was detected in $58 \%$ and $9.6 \%$ of total wounds at weeks 4 and 12 respectively, from the start of antioxidant dressing (Figure 5). At weeks 8 and 12, the same two wounds presented persistent biofilm presence (neuropathic and arterial etiology), nevertheless both wounds din not stop healing progression and showed a good evolution overtime.

The following cases show representative histories and evolutions with antioxidant dressing in three patients with chronic wounds from different etiologies.

\section{Case 1 - Pressure ulcer}

Figure 6 shows a 57-year-old patient with tetraplegia, kidney failure, hypertension, lymphedema and diabetes. Patient presented 


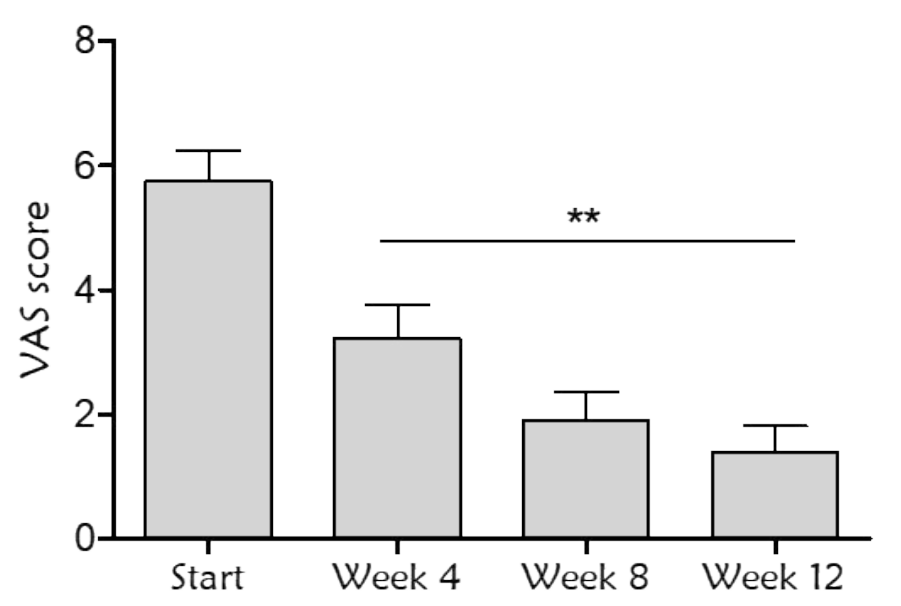

Figure 4. Pain assessment by Visual Analog Scale (VAS) determination during the treatment with the antioxidant dressing. Statistically significant differences were found with respect to the start of the treatment at all time points (Friedman test, $\mathrm{p}<0.0001$ ), and when comparing week 4 with respect to week $12(* * \mathrm{p}=0.008)$

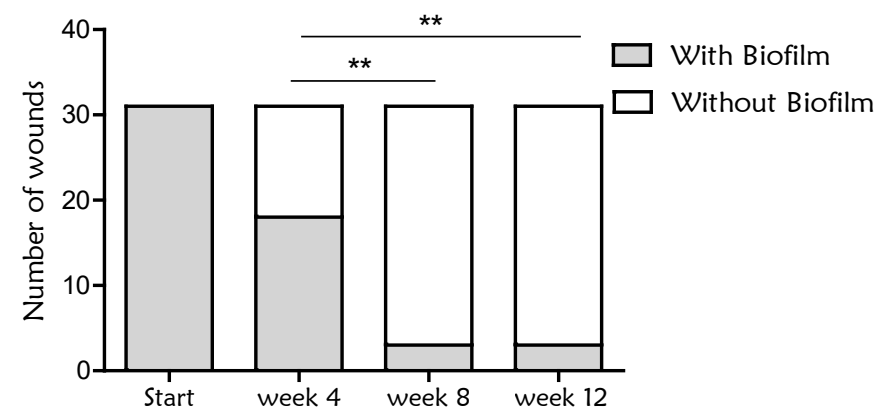

Figure 5. Presence of biofilm in the wound bed. Number of wounds with biofilm, determined following clinical observation (algorithm developed by Metcalf et al). It was observed a significant decreased of biofilm presence during the treatment with antioxidan dressing (Chi-square, $\mathrm{p}<0.0001$ ). Significant differences were also found when comparing week 4 with respect to week 8 and 12 (Chi-square, $* * p=0.001)$ a pressure ulcer category IV, located at right ischium, cavitated and with 25 months of evolution. The wound had recurrent infection and was stalled in the inflammatory phase. Wound bed presented sloughy tissue and biofilm. At week 8 , the treatment with antioxidant dressing achieved $74 \%$ of wound closure rate, significantly contributed to elimination of biofilm and devitalized tissues, and induced granulation tissue formation. At week 12, 89\% wound healing rate was achieved and final closure was reached at week 20.

\section{Case 2 - Calcifilaxys wound}

Figure 7 shows a 68-year-old woman with chronic renal failure (previous renal transplantation), secondary hypertension and deterioration of skin integrity among others comorbidities. She presented a calciphylaxis ulcer with 4 months of evolution located at outer third middle of the left leg. Wound had eversion in edges, moderate level of exudates, sloughy tissue, signs of local infection and severe pain (VAS 9/10). Treatment with antioxidant dressing dramatically reduced pain to $5 / 10,3 / 10$ and $0 / 10$ at 4,8 and 12 weeks from treatment start, respectively. Wound size reduced $73 \%$ at week 4 of treatment and wound closure was achieved at week 12.

\section{Case 3 - Venous leg ulcer}

Figure 8 shows an 82-year-old woman with vascular insufficiency, valve disease, poorly controlled anticoagulant treatment (Acenocoumarol), hypertension and chronic renal insufficiency. Patient suffered a leg trauma that produced a non-absorbable hematoma, previously treated with TPN. At the start of antioxidant treatment, ulcer was 6 months in evolution and was considered as a venous leg ulcer with a traumatic origin with presence of unhealthy granulation tissue in the wound bed, compatible with local signs of infection and presence of biofilm. Treatment with antioxidant dressing decreased patient pain from $7 / 10$ to $2 / 10$ at week 12 of treatment and a $97 \%$ of wound closure was obtained at the end of the study.
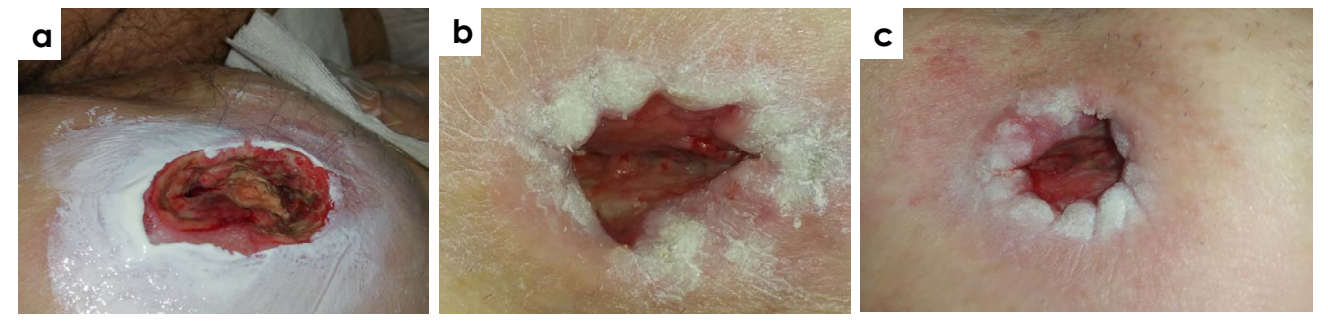

Figure 6. Case 1. Pressure ulcer category IV of 25 months of evolution in a 57-year old patient located on the ischium. Representative images at the beginning of the treatment (a) and evolution at week 8 (b) and week 12 (c) are shown. A wound closure rate of $74 \%$ and $89 \%$ was obtained at week 8 and week 12 of treatment with antioxidant dressing, respectively
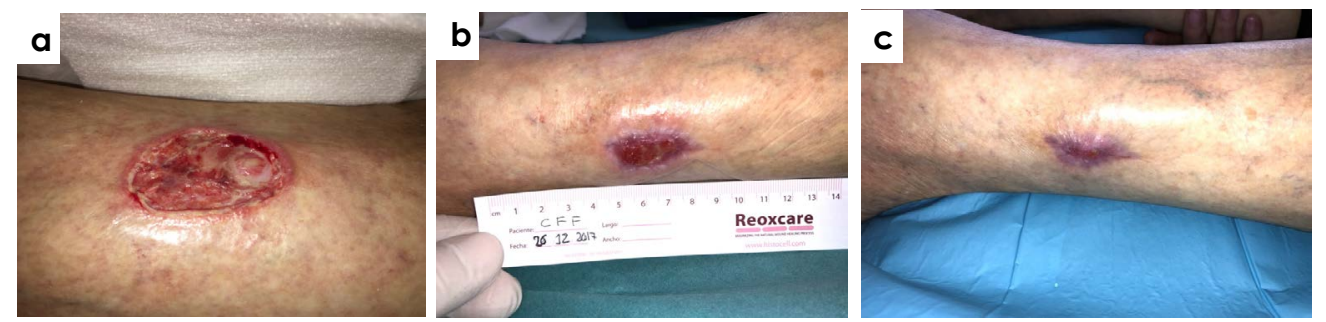

Figure 7. Case 2. Calciphylaxis ulcer in a 68-years old patient with 4 months of evolution. Representative images at the begining of the treatment (a) and evolution at week 8 (b) and week 12 (c) are shown. The healing process was stalled with severe pain and presence of devitalized tissue. It has been observed a significant evolution of wound healing with a $96 \%$ reduction of wound size at week 8 and final closure was already achieved at week 12 of treatment 

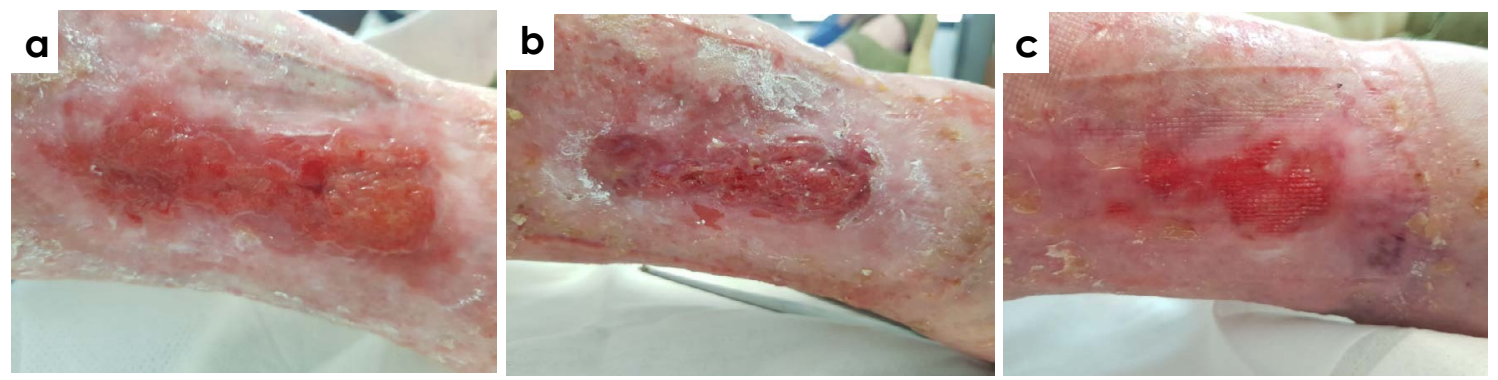

Figure 8. Case 3. 82-year old patient with a 6-months venous leg ulcers. Images show the evolution of the treatment after antioxidant wound dressing at the beginning (a), at week 4 (b) and week 8 (c). Wound size decreased $97 \%$ at the end of the study and a significant relief in wound pain was achieved, from 7/10 to 2/10 in VAS scale after 12 weeks of treatment

\section{Discussion}

One of the principal aims of Wound Care Specialists in Andalusia Public Health System (Spain) is to lead and promote research in chronic wound area and generate new protocols for wound care local practitioners in order to improve patient quality of live. This work presents a cases report about the application of a new antioxidant treatment indicated to the inflammatory phase of non-healing wounds. Patients included in this work were referred from 9 different primary care centers to chronic wound care specialist who coordinated the new treatment application and evaluated wounds evolution. All wounds were considered as non-healing wounds, as they complained with inclusion and exclusion defined criteria.

Most of patients exhibited recurrent wounds, with presence of soft devitalized tissues, and biofilm was detected in all of wounds according to clinical identification algorithm developed Metcalf et al. [20,21]. Some of the wounds also showed an important bacterial burden with signs of local infection $[21,22]$. All ulcers had not responded to previous treatments based on good standards of care. These included, among others, the control of bacterial bioburden using debridement techniques if required, adequate management of exudates to avoid maceration and compressive therapy in leg ulcers if indicated [23]. This way, antioxidant bioactive wound dressing is indicated for these type of wounds, stalled in the inflammatory phase to active wound progression through the management of oxidative stress situation, one of the main factors that contribute to inflammation persistence in damaged tissues [24]. taking into account the characteristics of included wounds, a 12 -week follow-up was considered as adequate to determine product functionality, including wound assessments at 4,8 and 12 weeks after treatment with antioxidant dressing.

RESVECH 2.0 wound assessment tool was applied to determine the evolution of the healing process $[25,26]$. This method allows the simultaneous assessment of key parameters that indicate the progression towards healing. In this case, a significant decrease of RESVECH 2.0 was observed with the treatment of antioxidant dressing, especially at the first 4 weeks of treatment, pointing out the role of this treatment in the first inflammatory phase of wound healing. Furthermore, at week 12 healing rate was $71 \%$, and $50 \%$ of the wounds totally healed. The achieved healing rate in this study can be considered favorable with respect to previous studies that pooled healing rates in chronic wounds and estimated an average complete closure rate of 31\% (8-20 weeks) or $10 \%$ for chronic venous leg ulcers (27 weeks) $[27,28]$.

Pain is a common factor in patients that present chronic wounds $[29,30]$, and the increase in the level of pain is related with wound stage and treatment procedures (inflammation, selection of the dressing, type of debridement, presence of infection or use of unsuitable products).
These factors cause a continuous state of poor quality of life in patients [31-33]. In this case, treatment with antioxidant bioactive dressing has been associated with the progression from inflammatory to proliferative phase of wound healing, which leads to significant decrease in pain (VAS scale measurements) and improvement in patient welfare.

On the other hand, it is reported that the presence of biofilm may be assumed in all chronic wounds [34]. Apart from following clinical recommendations to the control of biofilm, that rely on appropriate debridement [35-37], the synergic action of components of antioxidant dressing could prevent and contribute to eliminate biofilm [38]. Galactommanan present in absorbent matrix can have a physical effect over biofilm layer and acetylcistein and curcumin, have been described to have anti-biofilm activity [39-41]. In this study, deep cleaning with debridement if possible in each dressing change, in addition to antioxidant dressing, achieved elimination of biofilm in $90 \%$ of total wounds at 12 weeks of follow-up assessed by clinical observation. Results showed that antioxidant dressing could at least avoid biofilm interference in wound evolution. Further analyses are being conducted to determine the specific mechanisms through which the antioxidant dressing interacts with biofilm, using specific in vitro techniques that allow direct visualization and determination of wounds biofilm [42].

\section{Conclusion}

The study performed by chronic wound care specialists from Andalusia public Health System (south of Spain) concluded that antioxidant wound treatment is a suitable therapeutic alternative to achieve wound activation of non-healing wounds. The application of the treatment to complex wounds of different etiologies, resulted in a significant progression of healing evolution (improvement in wound closure rate and RESVECH 2.0) and pain relief. In addition, antioxidant dressing contributed to biofilm control in combination with deep cleaning of wounds in each dressing change. Furthermore, dressing demonstrated good performance for daily clinical practice and was very well tolerated by patients.

\section{Declarations}

Funding: We declare that we have received funding through the research project funded in the open and permanent call for projects to be carried out. Research and Innovation in the field of Primary Care of the Andalusian Health Service, with exp. No. AP-0138-2017, entitled: "Effectiveness of the advanced practice nurse, in the care of complex chronic wounds in Andalusia.

Acknowledgment: The authors wish to express their gratitude to all the nurses who have participated in the collection of data and in the follow-up of the patients, for being key investigators in the development 
of new clinical evidence. To all the patients who made this study possible.

Conflicts of interest: The authors declare no conflict of interest. The funders had no role in the design of the study; in the collection, analyses, or interpretation of data; in the writing of the manuscript, or in the decision to publish the results.

\section{References}

1. Evans K, Paul JK (2019) Overview of treatment of chronic wounds

2. Childs DR, Murthy AS (2017) Overview of wound healing and management. Surg Clin North Am 97: 189-207. [Crossref]

3. Frykberg RG, Banks J (2015) Challenges in the treatment of chronic wounds. $A d v$ Wound Care 4: 560-582. [Crossref]

4. Schäfer M, Werner S (2008) Oxidative stress in normal and impaired wound repair. Pharmacol Res 58: 165-171. [Crossref]

5. Vermeij WP, Backendorf C (2010) Skin cornification proteins provide global link between ROS detoxification and cell migration during wound healing. PLoS One 5: e11957. [Crossref]

6. Wagener FA, Carels CE, Lundvig DM (2013) Targeting the redox balance in inflammatory skin conditions. Int J Mol Sci 14: 9126-9167. [Crossref]

7. Kunkemoeller B (2017) Redox signaling in diabetic wound healing regulates extracellular matrix deposition. Antioxid Redox Signal 27: 823-838. [Crossref]

8. Madamanchi NR (2005) Oxidative stress and vascular disease. Arterioscler Thromb Vasc Biol 25: 29-38. [Crossref]

9. Ortega MA (2019) Patients with incompetent valves in chronic venous insufficiency show increased systematic lipid peroxidation and cellular oxidative stress markers. Oxid Med Cell Longev 2019: 5164576. [Crossref]

10. El Assar M (2020) Frailty as a phenotypic manifestation of underlying oxidative stress. Free Rad Biol Med 149: 72-77. [Crossref]

11. McMurray F (2016) Reactive oxygen species and oxidative stress in obesity-Recent findings and empirical approaches. Obesity 24: 2301-2310.

12. Roy S, Khanna S, Nallu K, Hunt TK, Sen CK (2006) Dermal wound healing is subject to redox control. Mol Ther 13: 211-220. [Crossref]

13. Juan Francisco JG (2019) The effectiveness of advanced practice nurses with respect to complex chronic wounds in the management of venous ulcers. Int J Environ Res Public Health 16: 5037. [Crossref]

14. Castro B, Palomares T, Azcoitia I, Bastida F, Del Olmo M, et al. (2015) Development and preclinical evaluation of a new galactomannan-based dressing with antioxidant properties for wound healing. Histol Histopathol 30: 1499-1512. [Crossref]

15. Castro B, Bastida FD, Segovia T, López-Casanova P, Soldevilla JJ, et al. (2017) The use of an antioxidant dressing on hard-to-heal wounds: A multicentre, prospective case series. J Wound Care 26: 742-750. [Crossref]

16. Edwin Kremer E, Atkinson H, Ignelzi R (1981) Measurement of pain: Patient preference does not confound pain measurement. Pain 10: 241-248. [Crossref]

17. Renner R, Seikowski K, Simon JC (2014) Association of pain level, health and wound status in patients with chronic leg ulcers. Acta Derm Venereol 94: 50-53. [Crossref]

18. Lunderberg TCM, Erikson SV, Malm M (1992) Electrical nerve stimulation improves healing of diabetic ulcers. Ann Plast Surg 29: 328-331.

19. Metcalf DG, Bowler PG, Hurlow J (2016) A clinical algorithm for wound biofilm identification. Acta Med Croatica 70: 73-79. [Crossref]

20. Steven LP, Claudia V, Gianfranco D, Benjamin AL (2015) Biofilms and wounds: An identification algorithm and potential treatment options. Advances in wound care 4: 389-397. [Crossref]

21. International Wound Infection Institute (IWII) Wound infection in clinical practice. Wounds International 2016.
22. Lloyd Jones M (2018) Series 3. Wound infection 3.1: The wound infection continuum explained. Br J Healthcare Assis12: 5.

23. World Union of Wound Healing Societies (WUWHS), florence congress, position document. Advances in wound care: The triangle of wound assessment. Wounds International 2016.

24. Cano Sanchez M (2018) Targeting oxidative stress and mitochondrial dysfunction in the treatment of impaired wound healing: A systematic review. Antioxidants 7: 98 [Crossref]

25. González de la Torre H, Quintana-Lorenzo ML, Perdomo-Pérez E, Verdú J (2017) Correlation between health-related quality of life and venous leg ulcer's severity and characteristics: A cross-sectional study. Int Wound J 14: 360-368. [Crossref]

26. Folguera-Álvarez C, Garrido-Elustondo S, Verdú-Soriano J, García-García-Alcalá D, Sánchez-Hernández M, et al. (2016) ECAMulticapa: Effectiveness of double-layered compression therapy for healing venous ulcers in primary care: A study protocol. $B M C$ nursing 15: 58. [Crossref]

27. Formentini M, Amante H, Fernandes LP (2014) Factors that influence healing of chronic venous leg ulcers: A retrospective cohort. An Bras Dermatol 89: 414-422. [Crossref]

28. Münter KC, Meaume S, Augustin M, Senet P, Kérihuel JC (2017) The reality of routine practice: A pooled data analysis on chronic wounds treated with TLC-NOSF wound dressings. J Wound Care 26: S4-S15. [Crossref]

29. Frescos N (2018) Assessment of pain in chronic wounds: A survey of Australian health care practitioners. Int Wound $J$ 15: 943-949.

30. Linares JP, Curto PM, Verdu J, Pla E (2014) El dolor en las heridas crónicas de las extremidades inferiores. $\operatorname{AgInf} 72: 175-177$.

31. Newbern S (2018) Identifying pain and effects on quality of life from chronic wounds secondary to lower-extremity vascular disease: An integrative review. Adv Skin Wound Care 31: 102-108. [Crossref]

32. Tilley C, Lipson J, Ramos M (2016) Palliative wound care for malignant fungating wounds: Holistic considerations at end-of-life. Nurs Clin North Am 2016: 513-531. [Crossref]

33. Fauziyah H, Gayatri D (2018) Pain, stress, and sleep quality in chronic wound patients Enferm Clin 28: 176-179. [Crossref]

34. Bjarnsholt T, Eberlein T, Malone M, Schultz G (2017) Management of wound biofilm made easy. London: Wounds international 2017.

35. Scalise A, Bianchi A, Tartaglione C, Bolletta E, Pierangeli M, et al. (2015) Microenvironment and microbiology of skin wounds: The role of bacterial biofilms and related factors. Semin Vasc Surg 28: 151-159. [Crossref]

36. Bianchi T, Wolcott RD, Peghetti A, Leaper D, Cutting K, et al. (2016) Recommendations for the management of biofilm: A consensus document. $J$ Wound Care 25: 305-317. [Crossref]

37. Rajpaul K (2015) Biofilm in wound care. Br J Community Nurs Wound Care: S6, S8, S10-1. [Crossref]

38. Dhall S, Do D, Monika G, Dayanjan Shanaka W, Angela B, et al. (2014) A novel mode of chronic wounds: Importance of redox imbalance and biofilm-forming bacteria for establishment of chronicity. PLoS ONE 9: e109848. [Crossref]

39. Rasmussen K, Nikrad J, Reilly C, Li Y, Jones RS (2016) N-Acetyl-1-cysteine effects on multi-species oral biofilm formation and bacterial ecology. Lett Appl Microbiol 62: 30-38. [Crossref]

40. Packiavathy IA, Priya S, Pandian SK, Ravi AV (2014) Inhibition of biofilm development of uropathogens by curcumin - An anti-quorum sensing agent from curcuma longa Food Chem 148: 453-460. [Crossref]

41. Finnegan S, Percival SL (2015) EDTA: An antimicrobial and antibiofilm agent for use in wound care. Adv Wound Care 4: 415-421. [Crossref]

42. Gregory S. Schultz, Dot W, Qingping Y (2015) Effectiveness of a monofilament wound debridement pad at removing biofilm and slough: ex vivo and clinical performance. $J$ Wound Care 27: 80-90. [Crossref

Copyright: (C2021 Jiménez-García JF. This is an open-access article distributed under the terms of the Creative Commons Attribution License, which permits unrestricted use, distribution, and reproduction in any medium, provided the original author and source are credited. 